\title{
Laser Therapy In Drug Induced Gingival Hyperplasia- A Case Report
}

\author{
Case Report \\ Volume 1 Issue 2- 2021 \\ Author Details \\ Sumit Garg, ${ }^{1}$ Rohit Chopra, ${ }^{2}$ Shivani Gupta, ${ }^{5}$ Rajesh Kumar Gupta, ${ }^{3 *}$ Preety Gupta \\ ${ }^{1}$ Department of Periodontics Guru Nanak Dev Dental College \& Research, India \\ ${ }^{2}$ Guru Gobind Medical College, India \\ ${ }^{3}$ Department of Oral Medicine and Radiology, India \\ ${ }^{4}$ Department of public health dentwastry, India \\ ${ }^{5}$ Department of Pedodontics Guru Nanak Dev Dental College \& Research, India \\ *Corresponding author \\ Rajesh Kumar Gupta, Reader in department of Oral Medicine and Radiology, Swami Devi Dyal Hospital and Dental College, \\ Barwala, India \\ Article History \\ Received: June 19, 2021 Accepted: June 28, 2021 Published: July 06, 2021

\begin{abstract}
Gingival excess is related with various components including innate infections, hormonal unsettling influences, helpless oral cleanliness condition, aggravation, neoplastic conditions, and unfavorable medication responses including anticonvulsants, calcium channel blockers, and immunosuppressants. This can have an inconvenient impact on the personal satisfaction and furthermore on high oral bacterial burden brought about by plaqueretentive regions. Different treatment modalities incorporate both careful (gingivectomy, periodontal fold, electrosurgery, and laser extraction) and nonsurgical methodologies. This case report reveals the treatment of drug induced gingival hyperplasia with laser.
\end{abstract}

\section{Introduction}

Gingival inflammation (recently called gingival hyperplasia or gingival hypertrophy) is portrayed by broadened gingival tissue with lobulated appearance that bit by bit stretches out along the labial, lingual, and coronal angles to cover the whole anatomic crown of teeth. It might frequently relate with torment and draining gums, which in cutting edge cases may cause obstruction with discourse, rumination, and aesthetics [1].

The American Academy of Periodontology characterized drugimpacted gingival expansion as "an excess or expansion in size of the gingiva coming about in entire or partially from foundational drug use." The drugs most generally coming about in gingival growth are antiepileptics (basically,phenytoin), immunosuppressants (fundamentally, cyclosporine), and calcium channel blockers (fundamentally, nifedipine and verapamil), among which phenytoin is the first and the most normally connected with gingival amplification [2].

\section{Case Report}

A 36-year-old female patient reported to the of department of Periodontology in Dashmesh Institute Of Reasearch and Dental
Sciences, Faridkot, India, with the chief complaint of swollen gums in both upper and, lower front jaw region since 2 years. History dated back to 2 years when patient first noticed swelling in her upper and lower front teeth region accompanied by bleeding on brushing which was occassionaly associated with local discharge. Patient underwent scaling for the same where she was advised with oral mouthwashes also. Patient got relieved for few days but since then patient is again experiencing the similar problem. Past medical records showed that the patient was hypertensive since the age of 25 , and from last 8 months, the patient had been put on amlodipine .Patient was moderately built, well nourished with normal gait and posture. Patient was comfortably seated and well oriented to time, place and surroundings. Patient was cooperative and answering to all questions. Extraoral examination didn't showed any significant finding. Intraoral examination revealed, generalised enlargement of gingiva in the region of maxillary and mandibular teeth was seen. Gingiva extends labially and palatally in the maxillary region and labially and lingually in the anterior mandibular region and bucally and lingually in the posterior mandibular region upto the occlusal third of the crown on the right side and middle third of the crown on the left side of upper and lower jaw without interfering in the occlusion. (Figure $1 \& 2$ ) The gingival tissue was lobulated and clefting was seen between each enlarged gingiva. No pus exudate was seen On palpation, inspectory findings were confirmed. The enlarged 
tissue was non-tender, soft and oedematous. There was presence of bleeding on probing. Based on clinical history and nearby assessment, temporary finding amlodipine prompted gingival enlargement was made. Complete hemogram values were inside as far as possible and Orthopantomogram reveals no hard bony changes.

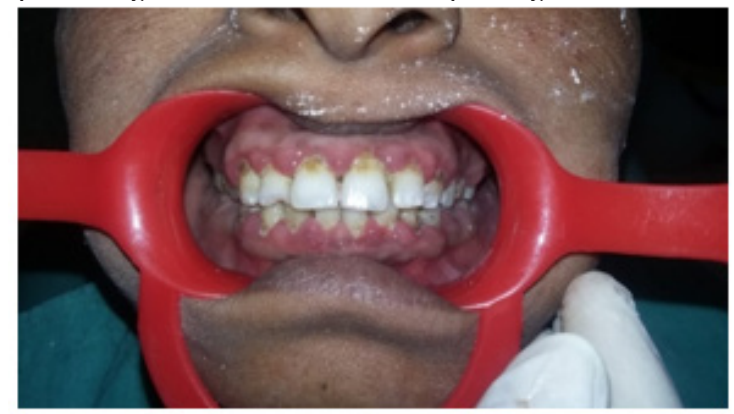

Figure 1: Left side of upper and lower jaw without interfering in the occlusion.

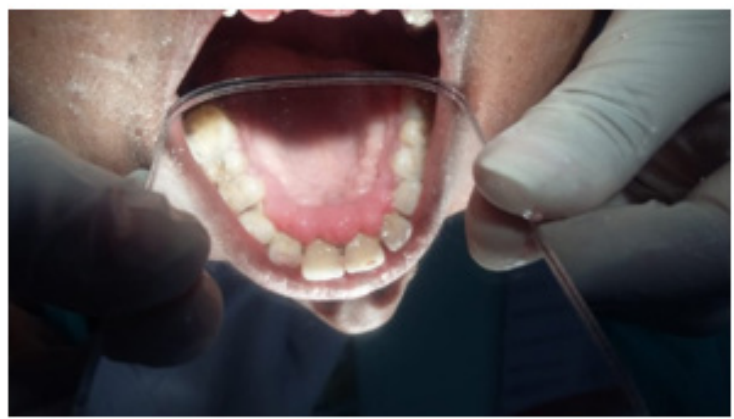

Figure 2: Left side of upper and lower jaw without interfering in the occlusion.

All the infected and inflamed tissue was taken out and Laser gingivectomy (Figure $3 \& 4$ ) was done utilizing delicate tissue diode laser, DCLase Dental Diode Laser 810-980nm (frequency 980nm) (Figure 5) having a $400 \mu \mathrm{m}$ breadth with an expendable tip with contact mode; power set at $2 \mathrm{~W}$ in nonstop beat was utilized Oral prophylaxis was performed and $0.2 \%$ chlorhexidine mouthwash $(10 \mathrm{~mL}$ bid for 7 days) was endorsed to the patient. The patient was told to keep up great oral cleanliness, and legitimate brushing strategies were disclosed to her. She was put on follow up of 3 weeks where decrease in gingival inflammation was seen especially in the upper jaw (Figure 6). The patient was endorsed with medicine and postoperative directions.

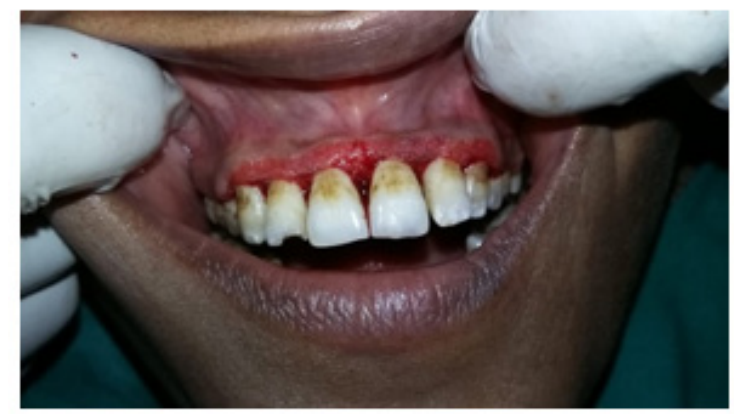

Figure 3: Infected and inflamed tissue was taken out and Laser gingivectomy.

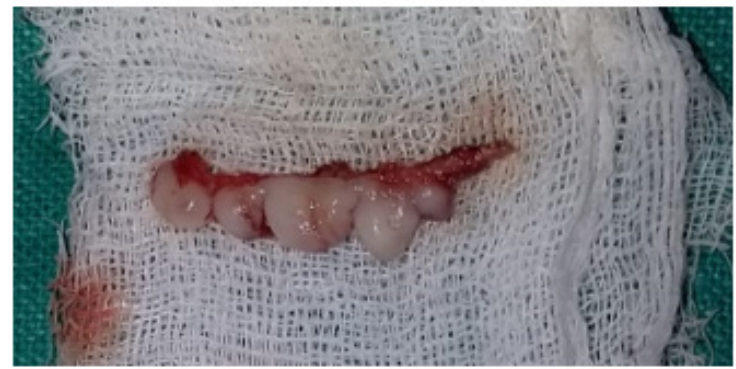

Figure 4: Infected and inflamed tissue was taken out and Laser gingivectomy.

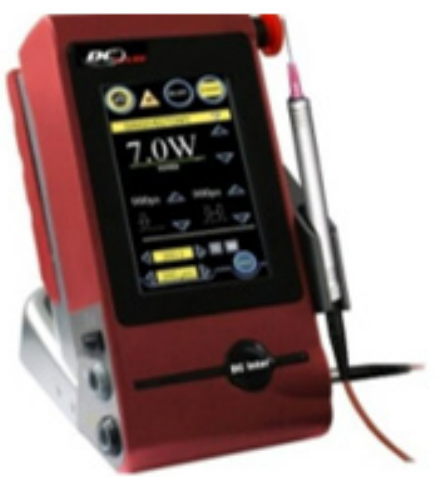

Figure 5: DCLase Dental Diode Laser 810-980nm (frequency 980nm).

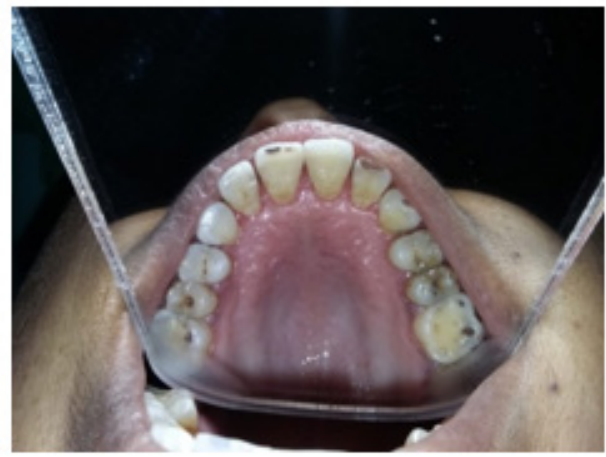

Figure 6: Decrease in gingival inflammation was seen especially in the upper jaw.

\section{Discussion}

In patients with hypertensive coronary illness the pervasiveness of gingival excess related with amlodipine is lower than that related with other calcium channel impeding specialists including nifedipine [3] Medication prompted gingival excess for the most part happens inside the initial 3 months of beginning medication treatment at a portion of $10 \mathrm{mg} /$ day and starts as an expansion of the interdental papilla. Albeit few instances of amlodipine-actuated hyperplasia have been accounted for, the current case is fascinating as it's anything but a low portion of amlodipine $(5 \mathrm{mg})$ and showed up on organization for a half year.

Seymour et al. [4] gave an audit on the pathogenesis of medication instigated gingival excess in which they thought about it's anything but a multifactorial model, including a collaboration of a few elements, which develops the association among medication and metabolite with the gingival fibroblasts. Inclining factors for these progressions are age, hereditary inclination, pharmacokinetic factors, drugactuated modifications in gingival connective tissue homeostasis, histopathology, ultrastructural factors and incendiary changes, and medication prompted activity on development factors [5].

The hidden component behind drug-instigated gingival hyperplasia includes incendiary and collagenase action because of diminished take-up of folic corrosive, blockage of aldosterone blend in adrenal cortex, and resulting criticism expansion in adrenocorticotropic chemical level and upregulation of keratinocyte development factor. Then again, aggravation may create because of direct harmful impacts of amassed drug in crevicular gingival liquid and additionally bacterial diseases. This irritation could prompt the upregulation of a few cytokine factors, for example, changing development factor- $\beta 1$ [7]

Numerous investigations have been directed which showed that amlodipine can't prompt gingival hyperplasia at $5 \mathrm{mg}$ once everyday portion regardless of whether taken for over a half year. It very well may be caused uniquely at a portion of $10 \mathrm{mg} /$ day [8]. The current case is novel in that even $5 \mathrm{mg} /$ day portion of amlodipine caused gingival hyperplasia following a half year of utilization. 
The system through which these medications incite gingival growth is still ineffectively comprehended. It has been discovered that phenytoin and calcium channel blockers repress the intracellular $\mathrm{Ca} 2+$ take-up consequently invigorating gingival fibroblasts. Not every one of the patients getting a similar medication foster gingival broadening. Conceivable explanation can be that people with gingival growth have fibroblasts with an unusual weakness to the medication. It has additionally been suggested that the vulnerability to pharmacologically instigated gingival augmentation might be represented by presence of differential extents of fibroblast subset in every person which display a fibrogenic reaction to these prescriptions. It has likewise been shown that the useful heterogenicity exists in gingival fibroblasts because of different improvements [9].

A synergestic improvement of collagenous protein blend by human gingival fibroblasts is discovered when these cells are presented at the same time to calcium channel blockers and raised degrees of interleukin- $1 \beta$ (a proinflammatory cytokine) in aggravated gingival tissues. Interleukin-6 likewise assumes a part in fibrogenic reactions of gingiva to these prescriptions. Interleukin- 6 targets fibroblasts which trigger the expansion of fibroblasts and apply the positive guideline on collagen and glycosaminoglycans union. So this cytokine has been proposed to assume a pathogenic part in fibrotic gingival amplification

As a treatment protocol, conservative periodontal measures are undertaken initially, which include vigorous gingival massage coupled with laser induced gingvectomy. However, when surgical measures are indicated, discontinuation of the drug is suggested. Drug-induced GO treated surgically shows recurrence but this can be decreased by proper home care, chlorhexidine gluconate rinses, and professional cleaning. Postsurgical recurrence rate varies in Drug-induced gingival enlargementfrom 3 to 6 months, but in most cases, the results are well maintained for 12 months.

\section{Conclusion}

Antihypertensive drug instigated gingival augmentation is brought about by undesirable impacts of foundational drug on the periodontal tissues. For a compelling control of this issue, appropriate treatment convention would be vital, which incorporates drug replacement and control of neighborhood fiery factors. Nonetheless, careful intercession is required when this succession of treatment neglects to determine the issue.

\section{References}

1. Nakib N, Ashrafi SS (2011) Drug-induced gingival overgrowth. Dis Mon 57: 225-230

2. American Academy of Periodontology. Glossary of Periodontal Terms. $4^{\text {th }}$ ed. Chicago, IL: American Academy of Periodontology 2001.

3. Triveni M G, Rudrakshi C, Mehta D S (2009) Amlodipine-induced gingival overgrowth. Journal of Indian Society of Periodontology 13(3): 160-163.

4. Seymour RA, Thomason JM, Ellis JS (1996) The pathogenesis of druginduced gingival overgrowth. J Clin Periodontol 23(3 Pt 1): 165-175.

5. Lafzi A, Farahani RMZ, Shoja MM (2006) Amlodipine-induced gingival hyperplasia. Medicina Oral Patología Oral y Cirugía Bucal 11(6): 480482.

6. Sonmez S, Cavdar C, Gunduz C, Nizam N, Biray C, et al. (2008) Do MMP-1 levels of gingival fibroblasts have a role in the gingival overgrowth of cyclosporine-treated patients? Transplantation Proceedings 40(1): 181-183.

7. Mavrogiannis M, Ellis J S, Thomason J M, Seymour R A (2006) The management of drug-induced gingival overgrowth. Journal of Clinical Periodontology 33(6): 434-439.

8. Pradhan S, Mishra P (2009) Gingival enlargement in antihypertensive medication. Journal of the Nepal Medical Association 48(174): 149-152.

9. Bagtzoglou A D (2004) Drug associated gingival enlargement. Journal of Periodontology 75(10): 1424-1431. 